\title{
Perspectivismo y objetividad en La genealogía de la moral
}

Perspectivism and Objectivity in On the Genealogy of Morality

Angel Rivera Novoa

Universidad de San Buenaventura-Bogotá, Colombia

Recibido: 24 de mayo de 2014

Enviado a pares: 24 de mayo de 2014

Aceptado por pares: 11 de agosto de 2014

Aprobado: 24 de septiembre de 2014

Pensamiento y Cultura | ISSN: 0123-0999 | eISSN: 2027-5331

pensam.cult | Vol. 17-2 | Diciembre de 2014 | pp. 47-72

DOI: 10.5294/pecu.2014.17.2.3 


\section{Perspectivismo y objetividad en La genealogía de la moral}

Resumen: En este artículo se analiza una tensión existente entre el objetivo que Nietzsche plantea en La genealogía de la moral y la tesis del 'perspectivismo' del mismo autor. Por un lado, Nietzsche afirma que su método llevará a dar cuenta de la historia de la moral realmente vivida; por otro, el perspectivismo sostenido por él implica que la objetividad de todo conocimiento es imposible. Para solucionar esta tensión se propone establecer una diferencia entre 'perspectivismo descriptivo' y 'perspectivismo prescriptivo'. Por último, se argumenta que lograr la objetividad propuesta por Nietzsche supone realizar una interpretación dominante en la que la voluntad de poder elimine otras interpretaciones.

Palabras clave: Nietzsche, La genealogía de la moral, perspectivismo, objetividad, interpretación, voluntad de poder.

\section{Perspectivism and Objectivity in On the Genealogy of Morality}

Abstract: In this article, it is analyzed an existent tension between the goal that Nietzsche established in On the Genealogy of Morality and Nietzsche's 'perspectivism' thesis. On one hand, Nietzsche claims that his method will allow him to account the history of moral truly lived. On the other hand, perspectivism implies that the objectivity of knowledge is impossible. I will propose to establish a difference between 'descriptive perspectivism' and 'prescriptive perspectivism' to solve the tension. Finally, I will argue that, to achieve the objectivity proposed by Nietzsche, it is necessary to make a dominant interpretation in which the Will of Power deletes others interpretations.

Keywords: Nietzsche, On the Genealogy of Morality, Perspectivism, Objectivity, Interpretation, Will of Power.

Según algunas afirmaciones de Nietzsche en el prólogo de su obra $L a$ genealogía de la moral, el método utilizado en dicha obra permitiría al autor dar una descripción fiel acerca "de la moral que realmente ha existido, de la moral realmente vivida" (GM, pról., 7). Estas afirmaciones pueden sugerir que el método genealógico de Nietzsche permite llegar -o por lo menos busca - una objetividad en tanto que su investiga- 
ción corresponde con la realidad. No obstante, esta pretensión parecería ser inconsistente con la idea nietzscheana según la cual todo conocimiento está sujeto al perspectivismo, razón por la cual, la objetividad en toda investigación sería imposible. En este artículo se propone hacer una lectura de algunos conceptos importantes de la obra de Nietzsche que permita hacer consistentes estas dos posturas. Para el propósito anterior, en primer lugar se expondrá la manera como Nietzsche comienza su investigación, a saber, advirtiéndonos sobre los propósitos y el método que empleará, además de establecer una crítica a los psicólogos ingleses de la moral justo antes de dar su visión acerca de las antítesis morales de 'bueno-malo (schlecht)' y 'bueno-malvado (böse)'. Lo anterior es importante, ya que se nos muestra qué tipo de investigación busca Nietzsche además de describir un ejemplo de cómo no es una investigación correcta (la investigación realizada por los ingleses). En segundo lugar, se dará cuenta de lo que Nietzsche entiende por 'perspectivismo' y cómo puede hacerse una lectura de la crítica de Nietzsche a los ingleses que descanse en esta tesis, de tal manera que el problema central sea abordado desde el inicio de la obra misma. En tercer lugar, propongo establecer una diferencia entre 'perspectivismo descriptivo' y 'perspectivismo prescriptivo', con lo cual no habría problema en afirmar que Nietzsche busca cierta clase de 'objetividad' (diferente a la objetividad como es entendida normalmente) que permite una consistencia de las dos posturas. Por último, se mostrará cómo dicha 'objetividad' solo es posible en tanto se haga una correcta 'interpretación' de los hechos a la manera como es expuesta en el tratado segundo.

\section{Propósito y método de La Genealogía}

En el prólogo a La genealogía de la moral, Nietzsche parece, por un lado, establecer cuáles son los objetivos centrales de su obra y cuál es el método que empleará para ello (cf. GM, pról. 2-7) y, por otro, tocar un par de temas que en principio parecerían estar aislados de lo anterior: una descripción de la naturaleza de 'las personas que conocen' como personas que no se conocen a sí mismos (cf. GM, pról. 1) y una advertencia a los lectores acerca de la manera de leer e 'interpretar' aforismos 
para poder lograr una buena comprensión de lo apuntado por él. En particular, Nietzsche exige que se hayan leído sus textos anteriores y que se posea el 'arte' del 'rumiar' como práctica fundamental en la aprehensión de aforismos (cf. GM, pról. 8).

Entre los apartados del 2 al 7 del prólogo de La genealogía de la moral, Nietzsche hace un recuento de lo que ha sido el problema de la moral para él. En ese sentido, lo que hace no es solo explicar el porqué de sus tesis, sino que, a la par, está haciendo una especie de autobiografía intelectual. En efecto, en principio nos dice que sus pensamientos acerca de la procedencia de las valoraciones morales tuvieron su primera aparición en Humano, demasiado humano, y que tales pensamientos son en esencia idénticos a los que se propone exponer ahora (cf. GM, pról. 2). Sin embargo, luego nos dice que sus pensamientos en ese entonces aparecieron en una edad inusual y que la pregunta planteada se refería a cuál era el origen exacto del mal, origen que entonces atribuyó a Dios. Con el paso de los años, y en el momento en que Nietzsche separa sus prejuicios teológicos de sus investigaciones filológicas, sumándole a esto un "aleccionamiento histórico y filológico", su problema fue transformado en el siguiente: “¿en qué condiciones se inventó el hombre esos juicios de valor que son las palabras bueno y malvado?, ¿y qué valor tienen ellos mismos?" (GM, pról. 3, subrayado por fuera del original).

Es interesante resaltar el hecho de que en esta etapa de su pensamiento - en la de La genealogía de la moral- Nietzsche ya presupone que el origen de la moral es el hombre mismo. Su pregunta está enfocada en la manera como el hombre inventó los valores y en cuáles fueron las condiciones en las que los estableció. Es notable también el hecho de que Nietzsche resalte y le dé importancia a la segunda pregunta (¿y qué valor tienen ellos mismos [los valores]? GM, pról. 3), pues como lo va a afirmar más adelante, la primera pregunta es una especie de medio (entre otros) para resolver esta última. Así pues, la cuestión que más le interesa a Nietzsche es aquella que tiene que ver con el valor de las valoraciones morales, las cuales nunca se han puesto en duda (cf. GM, pról. 6). Dice Nietzsche:

En el fondo lo que a mí me interesaba precisamente entonces era algo mucho más importante que unas hipótesis propias o ajenas acerca del 
origen de la moral (o más exactamente: esto último me interesaba sólo en orden a una finalidad para lo cual aquello es un medio entre otros muchos). Lo que a mí me importaba era el valor de la moral (GM, pról. 5).

Ahora bien, ¿qué quiere decir Nietzsche con el 'valor' de los valores morales? Pues bien, el autor parece darnos una pista en el apartado 3 del prólogo, justo después de enunciar la pregunta por el valor de los valores morales. Nietzsche se pregunta:

¿Han frenado o estimulado [los valores] hasta ahora el desarrollo humano? ¿Son un signo de indigencia, de empobrecimiento, de degeneración de la vida? ¿ $\mathrm{O}$, por el contrario, en ellos se manifiestan en plenitud, la fuerza, la voluntad de vida, su valor, su confianza, su futuro? (GM, pról. 3).

De esta forma, el valor de los valores parece determinarse por el hecho de si estos estimulan o no el desarrollo de la humanidad, si son signos de empobrecimiento e indigencia, o si son signos de la manifestación de la voluntad de vida. No obstante, el prólogo no nos dice nada acerca de lo que debe entenderse por cada uno de estos elementos (a saber, desarrollo humano, voluntad de vida, etc.). Tampoco nos dice nada acerca del porqué de la relación de las dos preguntas arriba mencionadas. Nos dice solo que la pregunta por el origen (que es la pregunta fundamental de La genealogía de la moral) es un medio para llegar al fin, que es la pregunta por el valor. No interesa en este artículo enfrentarse a estos dos vacíos que el prólogo nos deja; solo es necesario para el caso saber cuáles son los objetivos centrales de Nietzsche.

Luego de hablar de las preguntas fundamentales que pretende analizar, Nietzsche habla propiamente del método que utilizó para dilucidarlas. En efecto, en el numeral 7 del prólogo, Nietzsche comienza señalando que al abrírsele la perspectiva a propósito del problema de su investigación, empezó a buscar colegas y amigos (y hasta entonces los seguía buscando) que le ayudaran a plantear preguntas 'nuevas' en relación con los temas de la moral y — señala- más específicamente de "la moral que realmente ha existido, de la moral realmente vivida" 
(GM, pról. 7, énfasis mío). Entre estos colegas doctos que el alemán afirma haber buscado confiando en que su investigación lo llevaría a construir una metodología adecuada para encontrar buenas respuestas al problema, se encuentra Paul Rée.

No obstante, Nietzsche parece sugerir que fue precisamente en este punto donde se da una brusca separación entré él y Rée. Ya en el numeral 4 del prólogo, Nietzsche señalaba que Paul Rée con su libro El origen de los sentimientos morales lo impulsó a divulgar —a través de Humano, demasiado humano - por primera vez, sus pensamientos acerca de la moral. También señala que entonces se refirió a las tesis del mencionado libro no refutándolas, sino "poniendo, en lugar de lo inverosímil, algo más verosímil, y, a veces, en lugar de un error, otro distinto" (GM, pról. 4). De esta forma, es legítimo suponer que en principio Nietzsche estaba de alguna forma vinculado con las tesis del inglés; no obstante, esta apreciación puede ser apresurada. En el parágrafo 7 nos dice lo siguiente luego de preguntarse sobre la pertinencia de haber creído encontrar en Rée un verdadero compañero:

En todo caso, mi deseo era proporcionar a una mirada tan aguda y tan imparcial como aquélla una dirección mejor, la dirección hacia la efectiva historia de la moral, y ponerla en guardia, en tiempo todavía oportuno, contra esas hipótesis inglesas que se pierden en el azul del cielo. ¡Pues resulta evidente cuál color ha de ser cien veces más importante para un genealogista de la moral que justamente el azul; a saber, el gris, quiero decir, lo fundado en documentos, lo realmente comprobable, lo efectivamente existido, en una palabra toda la larga y difícilmente descifrable escritura jeroglífica del pasado de la moral humana (GM, pról. 7, subrayado fuera del original).

Luego de esto, Nietzsche afirma tajantemente que dicho pasado es totalmente desconocido para el inglés Rée dado que las investigaciones de este están viciadas por un corte darwinista, con lo cual se sustenta la afirmación según la cual, en este punto Nietzsche se separa de Rée -o por lo menos así lo expresa-. Ahora bien, el contraste que hace Nietzsche al respecto deja claro dos cosas: el análisis de Nietzsche no cae en la especulación floja y etérea propia de los psicólogos ingleses (de ahí 
la metáfora del azul de los ingleses) y, tal vez por esta misma razón, sus ideas acerca de la historia de la moral (las de Nietzsche) corresponden con la historia 'realmente vivida' y con 'la moral realmente existente'. Así lo ha afirmado Nietzsche varias veces en este mismo numeral. Lo anterior deja abierta la posibilidad de leer el método de Nietzsche como un método que busca una objetividad en términos realistas, esto es, se busca una objetividad en tanto que el gris al cual se refiere Nietzsche permite 'comprobar' la historia 'real' de la moral y en esa medida sus juicios corresponden claramente con los hechos. Con todo, y a pesar de que el problema de la objetividad será tratado en la siguiente sección de este artículo, hay que resaltar que es esta pretensión realista la que distingue a Nietzsche de Rée y de los ingleses, pues, en efecto, mientras los últimos no 'conocen' cuál es esa historia, Nietzsche asegura conocerla a través de los documentos, de lo 'realmente comprobable'.

Esta interpretación del mencionado numeral parece ser acuñada también por Nehamas (2002) quien en principio señala que la diferencia que marca Nietzsche con respecto a los planteamientos de Rée y de los ingleses consiste en que mientras el método de Nietzsche se basa realmente en la 'interpretación de textos', el de los ingleses no va más allá de una 'historia psicológica especulativa' (2002, 136-137). De esta manera, Nehamas dice:

[D] e hecho, en la primeras páginas de este libro, Nietzsche insiste una y otra vez en el argumento de que su genealogía, su interpretación de la historia y el valor de la moralidad tienen por objeto nada más y nada menos que la verdad; e insiste en que la suya, contrariamente a la de los otros, es la correcta (2002: 137, énfasis fuera del original).

También Berkowitz (2000) estaría de acuerdo con decir que la búsqueda de Nietzsche en La Genealogía de la moral es una búsqueda por la objetividad. También analizando el contraste entre Nietzsche y los ingleses, Berkowitz apunta:

En contraste con los ingleses, cuyos resultados están contaminados por su punto de vista, Nietzsche afirma haber descubierto las valoraciones morales y relaciones políticas originarias del hombre; supone 
osadamente que su propia mirada es nítida y cree firmemente que su punto de vista revela las cualidades básicas de los hombres y las verdaderas relaciones entre ellos $(2002,104)$.

Así pues, podemos afirmar - por lo menos desde el prólogo- que Nietzsche en efecto tiene cierta tendencia hacia la objetividad en su investigación. La interpretación de 'lo realmente comprobable' debe llevar a Nietzsche a una correspondencia entre sus tesis y la 'historia real de la moral'.

No obstante, el contraste con los ingleses no se reduce al prólogo; antes bien, los primeros apartados del primer tratado son dedicados a una crítica a los ingleses mucho más fuerte que la hecha en el prólogo. Allí, Nietzsche nos muestra cómo no es una investigación correcta. Así pues, lo primero que dice respecto a los psicólogos ingleses de la moral - ya fuera del prólogo- es que, por un lado, solo a ellos se les puede atribuir el único intento hasta entonces realizado de construir una genealogía de la moral y, por otra parte, que ellos aventajan en algo a sus libros toda vez que ellos presentan un enigma y, en esa medida, son ellos mismos el centro de interés de Nietzsche por encima de sus obras. El enigma del que habla Nietzsche consiste, de manera general, en la siguiente pegunta: “ $¿ q u e ́$ es lo que propiamente desean [los ingleses]?” (GM, I, 1). Ahora bien, esta pregunta se convierte en enigma en tanto los ingleses parecen, en toda investigación, aplicar una misma tarea e ir siempre en una misma dirección, la cual consiste en rescatar siempre la parte vergonzosa del hombre, esto es, aquello de lo cual el intelecto no desea que sea el origen de la moral, poniendo en estas cosas la parte realmente operante, por ejemplo, en la fuerza inercial, en el olvido o en el hábito. El enigma es entonces el hecho de que siempre apunten en la misma dirección. Nietzsche entonces se pregunta qué los motiva a eso. ¿Qué hay detrás de todo? A continuación, da posibles respuestas a modo de preguntas:

1 Esto es importante, en tanto es una de las dos posturas relevantes del presente artículo: el hecho de que Nietzsche busque objetividad en el estudio de la historia de la moral y, más aún, el hecho de que diga explícitamente que la posee. 
¿Es un instinto secreto, taimado, vulgar, no confesado tal vez a sí mismo, de empequeñecer al hombre? ¿O quizá una suspicacia pesimista, la desconfianza propia de idealistas desengañados, ofuscados, que se han vuelto venenosos y rencorosos? ¿O una hostilidad y rencor pequeños y subterráneos contra el cristianismo (y Platón), que tal vez no ha salido nunca más allá del umbral de la consciencia? ¿ O incluso un lascivo gusto por lo extraño, por lo dolorosamente paradójico, por lo problemático y absurdo de la existencia? (GM, I, 1).

Luego de señalar estos posibles motivos, a partir de los cuales los psicólogos ingleses de la moral encaminan sus investigaciones siempre en una misma dirección ${ }^{2}$, Nietzsche culmina su primer numeral afirmando que se le ha dicho que los ingleses son ranas viejas que se la pasan dando brincos en torno al ser humano y dentro de él como en su elemento propio, a saber, una ciénaga. Luego, Nietzsche nos dice, en un tono más bien irónico - para ser consistente con lo dicho en los dos numerales siguientes- que él no cree en eso, y desea con todo corazón, que el espíritu de aquellos ingleses sea valiente y magnánimo de tal forma que éstos sean capaces de sacrificar sus deseos y sentimientos a favor de la verdad (cf. GM, I, 1).

Pues bien, ya en el numeral segundo, Nietzsche se dispone a realizar la que, desde mi lectura, es la primera de dos críticas a los ingleses. Esta primera crítica consiste en decir que los historiadores ingleses carecen del espíritu histórico, i.e., ellos piensan de una manera a-histórica. ¿Por qué afirma Nietzsche que los ingleses carecen del espíritu histórico? Pues bien, él cree que la teoría inglesa acerca de la moral es insostenible históricamente y que su imprecisión histórica se evidencia desde el referente a partir del cual se trata de averiguar la procedencia del concepto 'bueno', pues los ingleses creen que 'bueno' tiene como referencia cualquier acción no egoísta llamada así por aquel a quién le era útil, esa utilidad

2 Berkowitz califica estos motivos arriba señalados como 'motivos despreciables' a diferencia de los motivos 'admirables' que según él, son los que Nietzsche expone al final de este primer numeral caracterizados todos ellos como el sacrificio por toda verdad por dura que esta sea (cf. Berkowitz 2000: 103). Los motivos despreciables estarían relacionados -afirma Berkowitz- directamente con el resentimiento. 
se olvida y por último debido al hábito aquellas acciones no egoístas siguieron siendo llamadas 'buenas,' "como si fueran en sí algo bueno" (GM, I, 2). Ahora bien, el espíritu de los ingleses es calificado como a-histórico debido a que, en realidad, la genealogía hecha por ellos no es más que el reflejo de su propia idiosincrasia inglesa (utilidad, olvido, hábito, y error), es decir, la extrapolación de su cultura a todas las existentes en la humanidad; más aún, de su cultura presente (utilitarismo del siglo XIX) al origen mismo de la humanidad. Acá cobra más sentido, la analogía hecha por Nietzsche acerca de las ranas. Esto es lo que precisamente hacen los ingleses, saltar alrededor y dentro del hombre como si este fuera su lugar habitual, su ciénaga. Es claro entonces, que la idiosincrasia de los psicólogos ingleses no corresponde a la historia universal de los orígenes de la palabra y el concepto 'bueno's. De esta manera, la primera crítica a los ingleses consiste en decir que su reconstrucción histórica es insostenible debido a que esta no es más que un reflejo de su idiosincrasia y, por lo tanto, su teoría no corresponde al verdadero origen de los conceptos morales ${ }^{4}$.

3 En este momento, Nietzsche expone cuál es — según él- la verdadera procedencia del concepto 'bueno'. Dicho concepto no se aplica a las acciones no-egoístas (el contraste de egoísta y no-egoísta nace justo cuando el primer uso de dicho concepto declina y el instinto de rebaño al fin puede llegar a hablar); en realidad, fueron los poderosos los que se adjudicaron a sí mismos el concepto 'bueno' en un pathos de la distancia, i.e., en un simple contraste con el plebeyismo, lo cual no es más que un contraste estamental y político, pero no nada tiene que ver esta adjudicación con la utilidad; esta, sugiere Nietzsche, no tiene relación con la nobleza, antes bien, hace parte del plebeyismo (por la sagacidad y cálculo que implica). Así pues, el origen real de la antítesis 'bueno' y ‘malo' es aquel sentimiento noble del pathos de la distancia que no marca más que una oposición radical entre castas y por lo tanto, no se aplica en principio a ninguna acción ni a la utilidad (cf. GM, I, 2). Nietzsche comienza a explicar su tesis con las siguientes palabras inmediatamente después de calificar a los ingleses como a-históricos: “[p]ara mí es evidente, primero, que esta teoría busca y sitúa en un lugar falso el auténtico hogar nativo del concepto «bueno»" (GM, I, 2) y empieza a señalar cuál es realmente el origen. Esto puede reforzar las pretensiones realistas de la investigación nietzscheana, pues por un lado afirma que la tesis inglesa es falsa e implícitamente nos está diciendo de nuevo que él posee el conocimiento de la historia real de la moral, de la moral realmente vivida.

4 En principio, es legítimo decir que aun cuando el inglés transpone su presente al pasado, esto no implica que el origen de las valoraciones no sea el adjudicado por los ingleses. Sin embargo, es evidente que Nietzsche no cree esto porque tiene una teoría diferente y, en segundo lugar, es conocido que Nietzsche opta por una imagen del mundo cambiante, por 
La segunda crítica a los ingleses viene a aparecer ahora en el numeral tercero, la cual nos dice básicamente que la teoría misma es inconsistente $-\mathrm{o}$ adolece de un contrasentido psicológico- - . El primer paso de la teoría inglesa nos dice que la utilidad de la acción no egoísta es el origen del concepto 'bueno' y que luego ese origen se habría olvidado. El paso de la utilidad al olvido es, para Nietzsche, algo inconcebible, pues la utilidad en ningún momento puede desaparecer de la consciencia; antes bien, debe apoderarse de ella cotidianamente ${ }^{5}$. Es más razonable - afirma Nietzsche - la teoría de Spencer, la cual nos dice que en principio 'útil' corresponde a 'bueno' e 'inútil' a 'malo' y esto ha sido así durante toda la historia; de tal forma, lo útil se presenta después como 'lo valioso en sí. La teoría es, en todo caso, falsa, pero por lo menos es consistente con la creencia según la cual la utilidad no puede devenir en el olvido (cf. GM, I, 3). Así, Nietzsche plantea estas dos críticas a los genealogistas ingleses de la moral (su teoría es insostenible históricamente y, además, es inconsistente) y, con esto, muestra entonces cómo no debe ser una investigación que pretenda hallar el origen de la moral.

\section{El perspectivismo y su paradoja}

Analizados ya los propósitos y el método de La genealogía de la moral, a través del prólogo de la obra y la crítica de Nietzsche a la genealogía de los ingleses, pasaremos a abordar la cuestión del 'perspectivismo', la cual, de entrada, parece no tener algún tipo de relación con lo hasta ahora expuesto. Ahora bien, un análisis exhaustivo del 'perspectivismo' superaría los límites de este escrito. Por lo tanto, y por los propósitos mismos, no serán examinados los argumentos que llevan a Nietzsche a tal posición, sino que se abordará la posición del 'perspectivismo' en sí misma, esto es, se examinará en qué consiste y cuáles son sus principales características.

lo cual, a través de la historia, los cambios de concepciones alteran las valoraciones mismas y para mirar el pasado no sería suficiente analizar las valoraciones del presente.

5 Para ser más precisos, habría que decir que la tesis de los ingleses es inconsistente con la creencia según la cual la utilidad no puede devenir en el olvido, pues la teoría inglesa bien puede rechazar dicha creencia y su genealogía no sería inconsistente. 
La tesis esencial del 'perspectivismo' consiste en decir que no hay un conocimiento objetivo del mundo, sino apenas múltiples interpretaciones del mismo. Apoyos a esta tesis pueden encontrarse desde el pensamiento temprano de Nietzsche, por ejemplo, en su temprano escrito Sobre verdad $y$ mentira en sentido extramoral (SVM). En dicho texto, Nietzsche nos plantea la idea de que la verdad no es la correspondencia del pensamiento con los hechos, sino apenas una metáfora, esto es, el hombre no accede a la verdad en sí, su verdad es un antropomorfismo en tanto que es más lo que allí hay del hombre que lo que hay de la realidad (cf. SVM, 25). La verdad, dice Nietzsche, no es buscada por sí misma, sino en virtud de sus consecuencias morales para la supervivencia social e implica siempre la construcción de un lenguaje arbitrario y, por lo mismo, de conceptos que ayudan a la denominación común de un grupo de personas a la realidad, creando así la diferencia entre verdad y mentira que, por supuesto, también sería arbitraria. En resumen, el pensamiento temprano de Nietzsche critica ideas clásicas de conocimiento tales como la verdad como correspondencia y la transparencia epistemológica: el conocimiento, al operar de una manera metafórica, no accede al campo de la 'verdad cristalina', y al ser impulsada por motivos morales no puede llegar a la objetividad: es apenas una perspectiva. Además de esto, no hay una búsqueda o un impulso a la verdad que sea desinteresado, sino que - por lo menos en sus inicios- la búsqueda de la verdad tiene fines políticos y morales a parte de la búsqueda misma.

Una de las formulaciones clásicas del 'perspectivismo' se encuentra en el aforismo 6 de Más allá del bien y del mal. Allí Nietzsche nos dice:

[L]as intenciones morales (o inmorales) han constituido en filosofía el auténtico germen vital del que ha brotado siempre la planta entera. [...] Yo no creo que, por tanto, un «instinto de conocimiento» sea el padre de la filosofía, sino que, aquí como en otras partes, un instinto diferente se ha servido del conocimiento. [...] Pues todo instinto ambiciona dominar: $y$ en cuanto tal intenta filosofar (MBM, 6).

Este pasaje nos ayuda a encontrar no el centro de la doctrina pero sí al menos su base: no hay una voluntad de conocimiento desintere- 
sado, sino que el conocimiento se mueve solo a partir de las intenciones, morales o no, del que conoce; por tal razón, el conocimiento no puede corresponder ni representar de manera fiel la realidad, sino que apenas es una perspectiva de la misma. Prejuicios morales, o incluso no morales, costumbres y vivencias pueden determinar el contenido de un conocimiento. Esto nos da una idea más clara a propósito del 'perspectivismo'.

En La ciencia jovial, Nietzsche presenta algunos aforismos donde nos expone algunas de las características principales del 'perspectivismo'. Así, por ejemplo, nos dice Nietzsche que 'los filósofos' están tan consagrados a la búsqueda de la verdad que se sumergen en "la gran pasión que inflama al que persigue el conocimiento, al que vive de continuo entre las tempestuosas nubes de los más arduos problemas y de las más pesadas responsabilidades [...] (y no es nada contemplativo, exterior indiferente, tranquilo, objetivo)" (CJ, 5, 351). Lo anterior - lo que está entre paréntesis - reafirma la definición dada anteriormente de 'perspectivismo', a saber, el hecho de que objetividad y 'perspectivismo' se contraponen. En efecto, aun cuando el filósofo crea buscar la verdad de manera desinteresada y contemplativa, esto en realidad no sucede. Antes bien, al embarcarse en una investigación, el filósofo no se da cuenta de que es literalmente movido por una moral específica que determina el rumbo de sus tratados (cf. MBM, 6). De este modo, su mirada no puede ser imparcial, i.e., no puede ser 'contemplativo', 'indiferente a los asuntos exteriores', 'tranquilo' no 'objetivo' y su investigación siempre va a estar guiada por sus instintos.

Por otra parte —en otro aforismo también de La ciencia jovial-, Nietzsche nos dice que al hombre se le abre un nuevo infinito, el de la posibilidad de la existencia de infinitas perspectivas e infinitas interpretaciones. Saber si la existencia posee algo antes ignorado o si una explicación sin razón es un sin-sentido, son exámenes que no pueden caer bajo el análisis científico más minucioso. "En tales análisis, el espíritu humano no puede hacer otra cosa que verse a sí mismo en sus propias perspectivas. Nos es imposible salir de nuestro ángulo visual” (CJ, 5, 374). Existe una curiosidad por saber qué otras clases de perspectivas existen o no. No obstante, está abierta la posibilidad para esto, de tal forma que la inmodestia acerca de la inteligencia humana como única 
productora de perspectivas es echada al suelo. "Por el contrario", dice Nietzsche, "el mundo se ha vuelto por segunda vez infinito para nosotros, por cuanto no podemos refutar la posibilidad de que sea susceptible de interpretaciones infinitas" (CJ, 5, 374).

De este modo, podemos sintetizar - si bien no todas- algunas de las características del 'perspectivismo': el 'perspectivismo' es la tesis según la cual es imposible que existan representaciones cognitivas que sean fieles a la realidad, i.e., no hay una correspondencia entre pensamiento y mundo, no hay objetividad ${ }^{6}$; lo anterior se da, entre otras cosas, por la influencia de los diversos instintos del hombre que guían las investigaciones. Estos instintos pueden ser morales o no-morales e incluso, toda una moral puede estar llevando a cabo cierta investigación o, en otras palabras, puede ser la base de la misma (cf. CJ, 5, 351); por esto, no existe alguna voluntad de conocimiento desinteresada. Quien conoce, por tanto, no puede ser contemplativo ni ajeno a lo externo (por lo que es legítimo suponer que existen instintos internos y externos, y que éstos últimos también impulsan el conocimiento). Por último, de un mismo objeto, pueden existir infinitas perspectivas, lo que prueba nuevamente que la objetividad queda fuera del campo del 'perspectivismo'.

Teniendo una visión del 'perspectivismo' según la cual todo conocimiento está sujeto a los intereses, intenciones y motivos de quien conoce, de tal forma que hay que renunciar a la objetividad, pasaré ahora a mostrar cómo la crítica de Nietzsche a los ingleses puede interpretarse a través de esta doctrina y, después de esto, dejar planteada la posibilidad de que la misma crítica (o parte de esta) que Nietzsche hace a los ingleses puede encaminarse a sus propias hipótesis acerca del origen de la moral.

Como se señaló, la crítica a los ingleses consiste básicamente en dos elementos: por una parte, es históricamente insostenible y, por otra, es en sí misma inconsistente (con la creencia según la cual lo útil deviene en

6 No debe leerse 'mundo' como 'cosa en sí propiamente. Para Kant puede haber objetividad sin que se pueda llegar a saber cómo es la 'cosa en sí. En esa medida, el realismo empírico (correspondencia o adecuación) es compatible con el idealismo trascendental. Así, se puede distinguir entre una representación objetiva de fenómenos y una no objetiva de los mismos, sin que esto suponga que la correspondencia se da entre pensamiento (o proposiciones) y 'cosa en sí. 
el olvido). Ahora bien, la primera parte de la crítica tiene dos premisas implícitas: 1) el origen que los ingleses adjudican al concepto bueno no corresponde a la historia real de la moral (cf. GM, I, 2) y 2) Nietzsche sí conoce cuál es el verdadero origen del mencionado concepto (cf. GM, pról. 7 \& GM, I, 2). Esto lleva a Nietzsche a sostener que los ingleses piensan de una manera esencialmente a-histórica; ¿cómo sustenta Nietzsche lo anterior? Pues bien, la primera premisa implícita parece descansar sobre el supuesto del 'perspectivismo', dado que el argumento de Nietzsche consiste en afirmar que el origen adjudicado por los ingleses (esto es, la deducción desde la utilidad hasta el error, pasando por el olvido y por el hábito) es simplemente una proyección de su idiosincrasia y de su moral actual, i.e., es una visión que no va más allá de su perspectiva. En efecto, la crítica parece descansar y reposar en el hecho de que no hay una objetividad en tanto su teoría de la historia de la moral es motivada por sus instintos morales, sus costumbres y su perspectiva. Esto puede llegar a ser más claro si se recuerda la metáfora de Nietzsche a propósito de las ranas: el inglés es una rana que da brincos alrededor y dentro del hombre (mira o trata de conocer al hombre) como si este (el hombre) fuera su hábitat fundamental, una ciénaga (como si este fuera una proyección de su perspectiva). Precisamente, en el numeral 1 del primer tratado de La genealogía de la moral, como se vio anteriormente, Nietzsche señala algunos motivos que impulsarían a los ingleses a emprender siempre la misma dirección. Vale la pena recordarlos:

¿Es un instinto secreto, taimado, vulgar, no confesado tal vez a sí mismo, de empequeñecer al hombre? ¿O quizá una suspicacia pesimista, la desconfianza propia de idealistas desengañados, ofuscados, que se han vuelto venenosos y rencorosos? ¿O una hostilidad y rencor pequeños y subterráneos contra el cristianismo (y Platón), que tal vez no ha salido nunca más allá del umbral de la consciencia? ¿O incluso un lascivo gusto por lo extraño, por lo dolorosamente paradójico, por lo problemático y absurdo de la existencia? (GM, I, 1).

Estos son los posibles motivos que los ingleses tendrían para ir siempre en la misma dirección, a saber, en la de sacar del hombre la 
parte más vergonzosa y darle el título de la más importante y la más operante: "en la vis inertiae [fuerza inercial] del hábito, o en la capacidad de olvido, o en una ciega y causal concatenación y mecánica de ideas" (GM, I, 1). Esta dirección es la ya descrita por Nietzsche en los numerales 2 y 3 . Ahora bien, ¿son estos los motivos que impulsa a los ingleses a ir en esa dirección junto con una moral propia? Vale la pena revisar lo dicho por Nietzsche en La ciencia jovial al respecto. Allí Nietzsche manifiesta que la moral no ha sido tomada como un problema real. Esto por la falta de individuos. Lo anterior es importante si se tiene en cuenta lo siguiente: los grandes problemas exigen un gran amor; para esto se necesitan individuos enérgicos y valerosos que sean capaces de mantener sus cimientos. Pero Nietzsche señala que hay una carencia de individuos, los pensadores parecen refugiarse en el problema tomándolo como sentido y fin. Otra cosa es acercarse a los problemas de manera impersonal, por una fría curiosidad. "[E]n este último caso no conseguirá nada" (CJ, 5, 345), ya que el problema resultará por consumirlos. La moral, por tanto, no ha sido un problema, no ha habido individuos que la enfrenten. Nietzsche señala haber creído encontrar en los ingleses sujetos que se enfrentaron al problema (esta misma creencia es expresada por Nietzsche en el prólogo a La genealogía de la moral, como se mostró anteriormente), pero esta creencia es falsa. Él sostiene que los ingleses "están ingenuamente al servicio de una moral determinada, de la que son portaestandartes y custodios" (CJ, 5, 345). El error de estos genealogistas es haber encontrado un consenso entre las costumbres de 'algunos' pueblos “ $y$ deducir de ahí una obligación absoluta, hasta para las relaciones entre individuos" (CJ, 5, 345).

De este modo, vemos cómo la crítica de Nietzsche a los ingleses genealogistas de la moral reposa en parte en la tesis del 'perspectivismo'. En efecto, la carencia del espíritu histórico se ve reflejada en el hecho de que los ingleses toman su perspectiva del asunto y le dan validez universal de una manera arbitraria. Además de esto, dichos genealogistas son portavoces de una moral altruista que se manifiesta en sus investigaciones. Por eso, la investigación de ellos no es objetiva, es falsa y está guiada por una serie de instintos que, como se vio en una nota al pie anterior, Berkowitz califica como despreciables. No quiero sugerir 
que la crítica es inválida. Lo que sí quiero sugerir es que el mismo supuesto que sustenta la crítica de los ingleses puede, a su vez, ser un supuesto de una crítica contra la teoría misma de Nietzsche. En efecto, si la doctrina del perspectivismo es correcta, Nietzsche no podría escapar de la misma y su visión acerca del origen de la moral estaría viciada por ciertos instintos (morales o no) que le harían perder a la teoría su objetividad pretendida según el prólogo de La genealogía.

Así, el análisis etimológico que hace Nietzsche de los conceptos de "bueno" y "malvado" y de "bueno" y "malo", puede estar viciado por su perspectiva del asunto al igual que su hipótesis sobre la metamorfosis conceptual y la rebelión de los esclavos. ¿Qué asegura la objetividad de la teoría nietzscheana del origen de la moral? ¿Qué asegura que Nietzsche, a diferencia de los ingleses, sí tenga el espíritu histórico que a los últimos les falta? Parece que Nietzsche puede entrar en una paradoja al estilo de Epiménides, el cretense, quien decía que "los cretenses son mentirosos" de tal forma que el valor de verdad del enunciado no se puede determinar debido a la autoreferencia. Lo mismo podría pasar con Nietzsche, quien diría: «todo es perspectiva, por lo cual no habría objetividad» y sería así imposible determinar el valor de verdad de la tesis nietzscheana. ¿Cómo asegurar que la teoría acerca del origen de la moral de Nietzsche tiene validez y corresponde efectivamente con la realidad? Más aún, ¿esto es lo que Nietzsche busca propiamente - a pesar de lo dicho por él en el prólogo—? ¿Es válida la crítica que se ha formulado de Nietzsche contra sí mismo? Para ir un poco más lejos: si no es posible construir una historia objetiva de la realidad, ¿cómo asegurar una crítica a la misma?

Reginster (2001) señala que sea cual sea la interpretación que se le dé al 'perspectivismo', se generará una paradoja de auto-referencia: "si toda visión está irremediablemente sometida a una perspectiva, ¿cómo puede Nietzsche defender visiones en ética y metafísica, $y$, más aún, cómo puede defender consistentemente el perspectivismo mismo?" $(2001,217)$. Esta paradoja tiene dos formas de ser resuelta (o existen dos propuestas de solución) según Reginster. Una solución es la de 'los dos niveles', según la cual, las tesis centrales acerca de ética, metafísica y del 'perspectivismo' mismo no entrarían bajo una mirada perspectivista. En 
esa medida, no existiría ninguna paradoja. No obstante, este argumento es ad hoc y no parecerían encontrarse indicios claros en la obra de Nietzsche para afirmar esto. La otra solución, es la 'solución perspectivista', según la cual, se tendría que aceptar que las tesis nietzscheanas —incluyendo la tesis del 'perspectivismo' - no van más allá de una perspectiva y están sujetas a ser reevaluadas. Pero aún si esto fuera así ¿por qué la tesis de los ingleses acerca de la moral es errónea a diferencia de la hipótesis de Nietzsche? ¿Qué es lo que en realidad las distingue?

\section{Perspectivismo prescriptivo y descriptivo: la interpretación como 'objetividad'}

En esta última sección, voy a defender la solución a la paradoja del 'perspectivismo' que Reginster llama 'solución perspectivista' proponiendo primero establecer una diferencia entre 'perspectivismo prescriptivo' y 'perspectivismo descriptivo' para culminar con la idea según la cual las pretensiones de objetividad de Nietzsche pueden ser consistentes con el 'perspectivismo' a través de la idea de 'interpretación'. Para lo anterior partiré del siguiente pasaje del tercer tratado de La genealogía de la moral:

A partir de ahora, señores filósofos, guardémonos mejor, por tanto, de la peligrosa y vieja patraña conceptual que ha creado un «sujeto puro del conocimiento, sujeto ajeno a la voluntad, al dolor, al tiempo», guardémonos de los tentáculos de conceptos contradictorios, tales como «razón pura», «espiritualidad absoluta», «conocimiento en sí»: -aquí se nos pide pensar en un ojo que de ninguna manera puede ser pensado, un ojo carente en absoluto de toda orientación, en el cual debieran estar entorpecidas y ausentes las fuerzas activas e interpretativas, que son, sin embargo, las que hacen que ver sea ver-algo, aquí se nos pide siempre, por tanto, un contrasentido y un no-concepto de ojo. Existe únicamente un ver perspectivista, únicamente un «conocer» perspectivista; y cuanto mayor sea el número de afectos a los que permitimos decir su palabra sobre una cosa, cuanto mayor sea el número de ojos, de ojos distintos que sepamos emplear para ver una misma cosa, tanto más completo será nuestro «concepto» de 
ella, tanto más completa será nuestra «objetividad». Pero eliminar en absoluto la voluntad, dejar en suspenso la totalidad de los afectos, suponiendo que pudiéramos hacerlo: ¿cómo?, ¿es que no significa esto castrar el intelecto? (GM III, 12).

Este pasaje parece hablar de 'perspectivismo' en dos sentidos diferentes: en la primera parte, nos habla de un 'perspectivismo descriptivo' -que es el 'perspectivismo' tal y como se analizó en la sección anterior-. Dicho perspectivismo sería la visión según la cual no existe ningún sujeto puro de conocimiento, i.e., el sujeto siempre alterará el contenido de la representación, no hay sujetos ajenos a la voluntad ni al tiempo. El diferente número de afectos de un sujeto (podemos decir sus instintos morales, no-morales, internos o externos) son las fuerzas 'activas e interpretativas' que dan una cierta orientación al ojo, esto es, hacen que ver sea ver algo. Sin estas afecciones es imposible - según este pasaje- la tarea del entendimiento. Por eso mismo, nadie, puede escapar a la doctrina del 'perspectivismo', esto es, nadie puede tener una imagen fiel de la realidad, ya que todo ver y todo conocer es perspectivista. Además, según lo dicho en el pasaje, no hay que decir que no existe una cosa fuera de nosotros, una realidad externa; antes bien, el 'perspectivismo' consiste en que hay muchas perspectivas 'de una misma cosa'. Esto es lo que denominaré 'perspectivismo descriptivo' que se puede resumir como la tesis de Nietzsche según la cual todos estamos sujetos a la perspectiva y, por lo tanto, no hay sujetos puros de conocimiento ni tampoco objetividad.

Ahora bien, aun cuando no hay una objetividad, Nietzsche afirma que sí existen grados de una cierta 'objetividad'. ¿Cómo pueden existir grados de una cosa que no existe? Pues bien, es notable el hecho de que Nietzsche - en el pasaje mencionado y en otros aforismos también del tercer tratado- utilicela palabra 'objetividad' entre comillas. Antes del pasaje citado, Nietzsche nos señala qué quiere decir con 'objetividad':

Ver alguna vez las cosas de otro modo, querer verlas de otro modo, es una no pequeña disciplina y preparación del intelecto para su futura «objetividad», -entendida esta última no como «contemplación desinteresada» (que, como tal, es un no-concepto y un contrasentido), 
sino como la facultad de tener nuestro pro y nuestro contra sujetos a nuestro dominio y de poder separarlos y juntarlos (GM, III, 12).

Vemos entonces cómo esta clase de 'objetividad', de la que Nietzsche nos dice que existen ciertos grados, es diferente a la objetividad normalmente entendida que supone una 'contemplación desinteresada. De tal forma, son dos objetividades distintas: en efecto, la primera, antes de eliminar los afectos e instintos del campo del conocimiento, los supone. En ese sentido, no por el hecho de que el 'perspectivismo descriptivo' sea cierto, debemos caer de inmediato en el escepticismo; antes bien, es posible llegar a un 'conocimiento perspectivista'. Justo en esto consiste el 'perspectivismo prescriptivo', i.e., dado que estamos sujetos a no ir más allá de nuestra perspectiva en nuestro conocimiento, debemos tratar de reunir la mayor cantidad de afectos, esto es, la mayor cantidad de ojos, para poder llegar a alcanzar una mayor 'objetividad'. La mayor 'objetividad' posible se da en cuanto más afectos y más perspectivas tengamos de una misma cosa. De todas formas, no son los afectos e instintos algo propiamente negativo, pero si se abandonan, ello significaría castrar el conocimiento humano.

También en Más allá del bien y del mal, Nietzsche nos muestra una idea similar a la que acá he denominado 'perspectivismo prescriptivo'. En el aforismo 34 de la mencionada obra Nietzsche nos dice en primer lugar que el hecho de valorar la verdad (digamos objetividad) como mejor que la mentira (digamos 'objetividad') no es más que un prejuicio moral y nada más. "[N]o existiría vida alguna — nos dice - a no ser sobre la base de apreciaciones y de apariencias perspectivistas" (MBM, 34). Con lo anterior, se reafirma la creencia según la cual un ver no perspectivista es un sinsentido. Si alguien intentara borrar el mundo de lo aparente, no quedaría ya nada de su «verdad». Luego de esto, Nietzsche nos dice:

¿[Q]ué es lo que nos fuerza a suponer que existe una antítesis esencial entre «verdadero» $\mathrm{y}$ «falso»? ¿No basta con suponer grados de apariencia y, por así decirlo, sombras y tonos generales, más claros y más oscuros, de la apariencia, - valeurs [valores] diferentes, para decirlo en el lenguaje de los pintores? ¿Por qué el mundo que nos concierne en algo - no iba a ser una ficción? (MBM, 34). 
Ya luego en el aforismo 36 de Más allá del bien y del mal, Nietzsche se pregunta si no es acaso algo lícito partir de la hipótesis según la cual lo único dado es lo pasional y lo instintivo $-\mathrm{y}$ no una realidad que se puede conocer objetivamente- y preguntarse si el mundo mecánico y material se puede comprender solo a partir de esto dado, es decir, de las pasiones e instintos (cf. MBM, 36). Es precisamente esto último el 'perspectivismo prescriptivo', dado que todo es una perspectiva $-\mathrm{y}$ lo único que nos es dado es el ámbito instintivo y pasional—, quien intente conocer debe jugar y poseer la mayor cantidad de instintos, pasiones y afectos para poder tener una visión más 'objetiva'.

Partiendo de esta diferencia entre perspectivismo 'descriptivo' y 'prescriptivo' hay que hacer la aclaración de que si lo dicho en la sección anterior a propósito de la crítica de Nietzsche a los ingleses es cierto, a saber, el hecho de que la crítica parece en parte reposar en el perspectivismo, no se puede decir que se basa en el perspectivismo descriptivo nada más. En efecto, Nietzsche no puede acusar a los ingleses por ver y conocer de manera perspectivista porque esta categoría de los que conocen así se extendería a todo el mundo (incluso a él mismo). La falta de los ingleses consistiría entonces en no tener un perspectivismo prescriptivo, esto es, en quedarse nada más en una perspectiva. En efecto, como se vio en el análisis del aforismo 345 de La ciencia jovial, los ingleses no son más que portavoces de una moral altruista y cristiana. El soporte de las investigaciones inglesas no va más allá de la perspectiva de esta moral, de su propia moral. Su instinto, por tanto, descansa solo en el hecho de ser idealistas desengañados y ofuscados. Es por esta razón por la que todas sus investigaciones van siempre en la misma dirección. No se dan cuenta de lo dicho por Nietzsche en el tratado tercero de La genealogía de la moral, a saber, que el conocimiento no está desligado de una voluntad y de un 'tiempo' y extrapolan su propio presente hasta el pasado. La crítica de Nietzsche consistiría entonces en el hecho de que los ingleses poseen solo una perspectiva, la perspectiva de una rana que cree que su ciénaga es el hombre mismo; por eso, los genealogistas ingleses no pueden tener un 'espíritu histórico'.

A partir de lo anterior, sabemos entonces que si la crítica de Nietzsche a los ingleses no consiste en decir que éstos ven y conocen de forma perspectivista, no es esta crítica entonces la que podría caer sobre el 
propio Nietzsche. El único riesgo que habría para que la crítica caiga sobre sí mismo es que Nietzsche no asumiera el 'perspectivismo prescriptivo'. No obstante, vale la pena anotar en este punto que la paradoja se ha tratado precisamente desde una 'solución perspectivista', esto es, si se parte de la hipótesis de que el perspectivismo (descriptivo) es cierto, cabe la posibilidad de que exista otra perspectiva según la cual el conocimiento es objetivo. No obstante, la paradoja se puede manejar solo en el momento en que se asume el 'perspectivismo descriptivo'; en otras palabras, no se le puede exigir al perspectivismo pretensiones de validez universal. El perspectivismo llega a ser válido si se asume, se trabaja e interpreta a partir de él. Ahora bien, ¿ le resta esto importancia a las tesis nietzscheanas? De ninguna manera, pues no hay que olvidar que existen diversos grados de 'objetividad'. De esta manera, lo que - se suponedistingue a la apreciación inglesa de la de Nietzsche es que la de él es más 'objetiva'. Así pues, es reconciliable la tesis del perspectivismo con la pretensión de Nietzsche en el prólogo de La genealogía de la moral de encontrar la historia real y efectiva de la moral, de la moral realmente vivida. Nietzsche, al tener un conocimiento 'perspectivista', no podría reflejar exactamente la verdad de la historia, pero sí puede representar la historia 'más objetiva', más verdadera. En ese sentido es en el cual Nietzsche busca la historia realmente vivida de la moral. Pretender una objetividad como se entiende normalmente, es decir, tener un amor a la verdad en sentido de correspondencia es abandonar precisamente el verdadero trabajo: "aquel querer-detenerse ante lo real, ante el factum brutum, aquel fatalismo de los petits faits (ce petit faitalisme como yo lo llamo) [...] aquel renunciar del todo a la interpretación (al violentar, reajustar, recortar, omitir, rellenar, imaginar, falsear, y a todo lo demás que pertenece a la esencia del interpretar)" (GM, III, 24).

\section{La interpretación como "objetividad" y conclusión}

Deseo terminar este artículo sugiriendo una última idea: 'tener más «objetividad» es interpretar de la mejor manera posible’. En el último pasaje citado al final de la sección anterior, se ve cómo la objetividad entendida clásicamente implica un detenimiento y justamente la negación del interpretar. 
Ahora bien, en el numeral analizado en esa misma sección se nos dice lo siguiente, a propósito de las fuerzas interpretativas:

[A]quí se nos pide pensar en un ojo que de ninguna manera puede ser pensado, un ojo carente en absoluto de toda orientación, en el cual debieran estar entorpecidas y ausentes las fuerzas activas e $\underline{\text { inter- }}$ pretativas, que son, sin embargo, las que hacen que ver sea ver-algo (GM, III, 12, énfasis fuera del original).

Y antes de esto Nietzsche nos había dicho:

Ver alguna vez las cosas de otro modo, querer verlas de otro modo, es una no pequeña disciplina y preparación del intelecto para su futura «objetividad», - entendida esta última no como «contemplación desinteresada» (que, como tal, es un no-concepto y un contrasentido), sino como la facultad de tener nuestro pro y nuestro contra sujetos a nuestro dominio y de poder separarlos y juntarlos: de modo que sepamos utilizar en provecho del conocimiento cabalmente la diversidad de las perspectiva y de las interpretaciones nacidas en los afectos. (GM, III, 12, énfasis fuera del original).

Así pues, se ve que la interpretación juega un papel importante cuando Nietzsche está hablando de 'perspectivismo prescriptivo'. En efecto, para que un ojo vea algo, esto es, para que haya un conocimiento, se necesita de fuerzas activas e 'interpretativas' las cuales surgen de los afectos mismos del sujeto que conoce. Así pues, es la interpretación la que da una dirección al conocimiento, esto es, la que da una perspectiva al conocimiento. De este modo, algo es 'más objetivo' que otra cosa, si es una interpretación más fuerte. Analizaré grosso modo qué entiende Nietzsche por 'interpretación' en el tratado segundo de La genealogía de la moral. Allí, Nietzsche está tratando el origen de la pena y en el numeral 12 se detiene para tratar el siguiente problema: no hay que confundir el origen de una cosa con su finalidad.

El problema del origen y el problema de la finalidad son pues dos problemas totalmente diferentes, aunque frecuentemente se les 
confunde. Los genealogistas - señala Nietzsche- han actuado de un modo muy ingenuo: buscan alguna finalidad propia de la pena (e.g. la venganza o la intimidación) y despreocupadamente identifican tal finalidad con el origen ${ }^{7}$. La finalidad es un proceso que se da sobre algo ya establecido, cuando algo se ha ya conformado, esto se 'interpreta' una y otra vez gracias a un poder que es superior, es decir, es transformado a una nueva utilidad o finalidad. Todo lo que acontece en el mundo orgánico es un enseñorearse y un subyugar, lo cual, a su vez, es un 'reinterpretar', un dar un nuevo sentido borrando el anterior. Así pues, donde hay una finalidad, hay una manifestación de que una voluntad de poder se ha enseñoreado y ha subyugado, i.e., ha interpretado (algo menos poderoso que aquella voluntad). De este modo, el desarrollo de algo no se da en virtud de una meta única; antes bien, el progreso de algo se da en la cadena de subyugaciones a las que se somete continuamente y de modo más bien casual. La interpretación es por tanto, en este contexto, la subyugación de una interpretación precedente y el apoderamiento (o enseñoramiento) de 'la realidad' para dotarla de un nuevo sentido o finalidad (cf. GM, II, 12) .

Así, si se tiene en cuenta que el vehículo que lleva al conocimiento a tener una determinada perspectiva es el acto interpretativo mismo, tendríamos que decir que cuando hay una mayor 'objetividad', esto es, cuando hay una mayor cantidad de perspectivas ( $\mathrm{y}$ con ellas, una mayor cantidad de instintos y afectos) es cuando se da una manifestación de una voluntad de poder que imprime un nuevo sentido a algo ya existente. De esta manera, podemos suponer, por ejemplo, que el objeto existente es la historia de la moral, y, en ese caso, la interpretación genuina se da

7 Seguramente Nietzsche se refiere de nuevo en este momento a los genealogistas ingleses - ¿ ¿ [c]ómo actúan, sin embargo, en este caso los genealogistas de la moral habidos hasta ahora?” (GM, II, 12, énfasis fuera del original)—. En esta medida, la crítica hecha en el primer tratado podría complementarse acá del siguiente modo: en su genealogía de la moral, los ingleses adjudican el origen de la valoración bueno a lo útil; sin embargo, el origen y la finalidad o utilidad no pueden ser confundidos.

8 Vale la pena tener en cuenta que Nietzsche está hablando acá no solo de documentos a interpretar, sino de todo el mundo orgánico. Así, cuando dentro de este mundo algo someta a otra cosa a través de una voluntad de poder más poderosa, hay un interpretar. Sin embargo, acá interesa nada más lo que tiene que ver con un ámbito epistemológico. 
cuando se logra imprimir un nuevo sentido a esa historia, sacrificando el sentido anterior que esta misma historia tenía. Es por esta razón que en uno de los aforismos de Más allá del bien y del mal que se revisaron en este artículo Nietzsche señala:

[L]as intenciones morales (o inmorales) han constituido en filosofía el auténtico germen vital del que ha brotado siempre la planta entera. [...] Yo no creo que, por tanto, un «instinto de conocimiento» sea el padre de la filosofía, sino que, aquí como en otras partes, un instinto diferente se ha servido del conocimiento. [...] [T] odo instinto ambiciona dominar: $\mathrm{y}$ en cuanto tal intenta filosofar (MBM, 6, énfasis fuera del original).

Dado que todo instinto desea dominar y son precisamente los instintos los que permiten la fuerza interpretativa en el acto de conocer (y entre ellos el más fundamental: la voluntad de poder), una genuina interpretación - y por tanto una 'objetividad mayor' - se da cuando se imprime un nuevo sentido a algo, cuando la voluntad de poder se enseñorea sobre ello. Así, si Nietzsche pretende que su investigación sea más 'objetiva', debe realizar una reinterpretación de la historia de las valoraciones morales que elimine las interpretaciones precedentes, i.e., donde estas sean sacrificadas.

\section{Bibliografía}

Nietzsche, Friedrich. 2000. Sobre verdad y mentira en sentido extramoral (SVM), Luis M. Valdés (trad.), Madrid: Ed. Tecnos.

Nietzsche, Friedrich. 1975. Genealogía de la Moral (GM), Andrés Sánchez Pascual (trad.). Madrid: Alianza Ed.

Nietzsche, Friedrich. 1997. Más allá del bien y del mal (MBM), Andrés Sanchez P. (trad.), Madrid: Alianza Editorial.

Nietzsche, Friedrich. 1996. Humano, demasiado humano, un libro para espíritus libres Vol. 1 (HDH), Alfredo Brotons (trad.). Madrid: Akal Ediciones.

Nietzsche, Friedrich. 1967. La voluntad de poder II, (VP), en Obras Completas. Buenos Aires: Aguilar. 
Nietzsche, Friedrich. 1985. La ciencia jovial (CJ), José Jara (trad.). Caracas: Monte Ávila Editores.

\section{Bibliografía Secundaria}

Berkowitz, Peter. 2000. Nietzsche: La ética de un inmoralista. María Condor (trad.). Madrid: Cátedra, Teorema.

Leiter, Brian. 1997. "Nietzsche and the morality critics" en Ethics, 107, No. 2: 250-285.

Meléndez, Germán. 2007. ¿(Auto-)superación de la moral como condición de su conocimiento? Ponencia presentada el 7 de febrero de 2007 en un seminario sobre La genealogía de la moral en la Universidad Nacional de Colombia- inédito).

Miggotti, Mark. 1998. "Slave Morality, Socrates, and the Bushmen: A Reading of the First Essay of On the Genealogy of Morals" en Philosophy and Phenomenological Research 58, No. 4: 745-779.

Nehamas, Alexander. 2002. Nietzsche. La vida como literatura, R. García Rodríguez (trad.), Madrid: Turner.

Nehamas, Alexander. 1983. "Immanent and Transcendent Perspectivism in Nietzsche", en Nietzsche-Studien, 12: 473-490.

Pickett, Brent.L. 1997. "Nietzsche's Ethics", en The journal of Politics, 59 No. 2: 576-582.

Reginster, Berdnard. 1997. "Nietzsche on Ressentiment and Valuation", en Philosophy and Phenomenological Research, 57 No.2: 281-305.

Reginster, Berdnard. 2001. “The Paradox of Perspectivism”, en Philosophy and Phenomenological Research, 62 No.1: 217-233.

Strong, T. 1985. "Text and pretext: reflections on Perspectivism in Nietzsche”, en Political Theory, 13, No. 2: 164-182. 